\title{
A Model-Free Control System Based on the Sliding Mode Control Method with Applications to Multi-Input-Multi-Output Systems
}

\author{
Agamemnon Crassidis, Fares El Tin \\ Rochester Institute of Technology \\ 76 Lomb Memorial Drive, Rochester, New York 14623, U.S. \\ alceme@rit.edu; fse5947@ rit.edu
}

\begin{abstract}
In this paper, a model-free sliding mode control technique for linear and nonlinear uncertain multi-input multi-output (MIMO) systems is proposed. The developed method does not require a mathematical model of the dynamic system. Instead, the controller relies on state measurements and estimates of the error between previous and current control inputs to stabilize the system. Knowledge of the system's order, shape of the control input matrix, and control gain bounds, if non-unitary, are the only variables required to develop the controller. Lyapunov's stability criterion is used in the derivation process to ensure closed-loop asymptotic stability. High frequency chattering, often observed with the sliding mode control method, is eliminated using a smoothing boundary layer. Simulations are performed on a nonlinear two mass-spring-damper system and a quadrotor model to examine the performance of the proposed control law.
\end{abstract}

Keywords: Model-Free, Sliding Mode Control, Multi-Input Multi-Output, Actuator Dynamics, Quadrotor

\section{Introduction}

In nonlinear control theory, sliding mode control is a robust control system capable of handling systems with modelling inaccuracies and outside disturbances. This technique is able to achieve perfect tracking in any system by reducing the tracking problem to a $1^{\text {st }}$-order stabilization problem [1]. In order to handle the uncertainties and disturbances that are present, a discontinuous term is introduced in the control input formulation. However, as a side effect of doing so, high frequency chattering of the control effort occurs. This undesirable phenomenon is resolved by introducing a smoothing boundary layer, while still achieving perfect control, and stability in the Lyapunov sense.

Due to its robustness, various sliding mode control (SMC) schemes have been developed for different applications. Runcharoon et al. [2] designed a PD controller for position and altitude control of a quadrotor, and combined that with SMC of the Euler angles. Xu et al. [3] proposed an approach to stabilize underactuated systems, systems with fewer inputs that outputs, using SMC. The system is transformed into cascade normal form, utilizing a systematic method proposed by Olfati-Saber [4], before being used in the design of the controller. The developed method was simulated on both a translational oscillator with rotational actuator (TORA) system and a quadrotor model.

The main hurdle to using SMC in controlling complex systems is the requirement of a well-defined mathematical model describing the behaviour of the system. Therefore, several researchers have investigated various types of model-free SMC in order to simplify the process. Munoz-Vazquez et al. [5] introduced a model-free integral SMC in combination with a passive velocity field (VF) to control a quadrotor. The VF was used to establish the desired path for the quadrotor in a certain environment, and the model-free integral SMC was used to drive the quadrotor to the desired path. Crassidis and Mizov [6 developed a model-free sliding mode controller which relies only on previous control inputs and state measurements to drive a system's states towards the desired trajectory. Crassidis and Reis [7] derived a similar controller to that introduced by Crassidis and Mizov in [6], but employed a different approach towards the formulation of the control input, while also requiring only previous control input and state measurements. The method was applied to linear and nonlinear single-input single-output (SISO) systems, with the presence of measurement noise.

The main contribution of this paper is to extend the work done by Crassidis and Reis in [7] into applications on MIMO systems, both fully-actuated and underactuated. The only characteristics of the system required to be known are the order of the system, shape of the input matrix, and an estimate of the control input gain bounds. The outline of this paper is as follows; following the introduction in Section 1, Section 2 will describe the system under control and introduce the sliding 
surface. Section 3 will develop the control law. Finally, Section 4 will simulate the controller on two $2^{\text {nd }}$-order nonlinear MIMO systems.

\section{System Description}

Consider the following $\mathrm{n}^{\text {th }}$-order autonomous system:

$$
x_{p}{ }^{n}=f_{p}(x ; t)+[B(x ; t)]_{p m} u_{m}
$$

where $p$ and $m$ are the \# of outputs and inputs, respectively, $x$ is the system states, $f(x ; t)$ defines the equations in $x$ and $t, B(x ; t)$ is a $p * m$ matrix of control input gains, and $u$ is the control input.

The system is redefined to the following:

$$
x_{p}^{n}=x_{p}^{n}+[B] u_{m}-[B] u_{m_{k-1}}-[B] u_{m}+[B] u_{m_{k-1}}
$$

where $u_{m_{k-1}}$ is the previous control input. An error parameter, $\varepsilon$, describing the error between the current control input $u_{m}$ and the previous control input $u_{m_{k-1}}$ is defined as:

$$
\varepsilon_{m}=u_{m_{k-1}}-u_{m}
$$

In order to compute the control law without encountering an algebraic loop throughout the simulation, an estimate of the control input error is defined as:

$$
\hat{\varepsilon}_{m}=u_{m_{k-1}}-u_{m_{k-2}}
$$

where $u_{m_{k-2}}$ is the previous control input of the previous control input. The control input error, although not exactly known, is assumed to be bounded by the following inequality:

$$
\left(1-\sigma_{l}\right) \hat{\varepsilon}_{m} \leq \hat{\varepsilon}_{m} \leq\left(1+\sigma_{u}\right) \hat{\varepsilon}_{m}
$$

where $\sigma_{l}$ and $\sigma_{u}$ are the lower and upper bounds, respectively, of the control input error estimate. At high sampling times, the error estimate will equal the actual error, thus the bounds will be approximately zero.

\subsection{Sliding Surface}

The sliding surface, as defined by Slotine et al. in [1], and extended into MIMO applications, is as follows:

$$
s_{m}=(d / d t+\lambda)^{n-1} \tilde{x}_{p}(t)
$$

where $\lambda$ is a strictly positive constant, defining the slope of the sliding surface, and $\tilde{x}_{p}(t)$ is the tracking error, $\tilde{x}_{p}(t)=x_{p}(t)-x_{p_{d}}(t)$.

Thus an $n^{\text {th }}$-order system has been reduced to $1^{\text {st }}$-order problem of keeping the scalar $s_{m}$ at zero, for all input and output paths.

In order to ensure that the system's states remain asymptotically stable during the reaching phase, while the controller drives the states towards the sliding surface, Lyapunov's direct method, describing a stable system as one with a decaying energy rate, will be used. The energy of the system will be defined using the following equation:

$$
V(x)=(1 / 2) s^{2}
$$

which is positive definite for all values of $s$. The rate of energy is obtained as: 


$$
\dot{V}(x)=s \dot{s} \leq 0
$$

Eq. (8) is then redefined as:

$$
\dot{V}(x)=-\eta|s| \leq 0
$$

where $\eta$ is a small positive constant, therefore negative-definiteness of Eq. (9) is guaranteed, and the closed-loop system is asymptotically stable.

\section{Model-free Sliding Mode Controller}

The model-free sliding mode controller requires a set of assumptions to be satisfied in order to proceed with the derivation of the control law. All system states must be both observable and controllable, since the controller relies on state measurements to drive the system towards the desired trajectories. Additionally, if the control input is not generated digitally, by a computer or microprocessor, it is assumed that it is measureable, to be used in the control law. Since both systems to be illustrated in a later section are $2^{\text {nd }}$-order, a $2^{\text {nd }}$-order sliding surface will be derived.

\subsection{Control Law for SISO and Square MIMO Systems}

The control law is obtained by differentiating Eq. (6) with respect to time and setting the equation to zero, to ensure states will remain on the surface once reaching it. For a $2^{\text {nd }}$-order system, this results in:

$$
\dot{s}_{m}=\ddot{\tilde{x}}_{p}+\lambda \dot{\tilde{x}}_{p}=\left(\ddot{x}_{p}-\ddot{x}_{d_{p}}\right)+\lambda\left(\dot{x}_{p}-\dot{x}_{d_{p}}\right)=0
$$

Substituting in Eq. (2) into Eq. (10) and solving for the control input results in the following:

$$
u_{m}=[B]^{-1}\left[-\lambda\left(\dot{x}_{p}-\dot{x}_{d_{p}}\right)-\left(\ddot{x}_{p}-\ddot{x}_{d_{p}}\right)\right]+u_{m_{k-1}}-\varepsilon_{m}
$$

In order to assure robustness of the controller against system uncertainties, a discontinuous term is added to the control input in Eq. (11) :

$$
u_{m}=[B]^{-1}\left[-\lambda\left(\dot{x}_{p}-\dot{x}_{d_{p}}\right)-\left(\ddot{x}_{p}-\ddot{x}_{d_{p}}\right)-\eta \operatorname{sgn}\left(s_{m}\right)\right]+u_{m_{k-1}}-\varepsilon_{m}
$$

where $\operatorname{sgn}(s)$ is the signum function.

Another clear constraint for Eq. (12) lies in the inverse of the control input gain matrix term, $[B]^{-1}$. To be able to compute the control input, the gain matrix needs to be invertible. However, this is only satisfied when the \# of inputs equals the \# of outputs, in fully-actuated systems, in which case the application of the model-free sliding mode control becomes similar to that proposed by Crassdis and Reis in [7].

\subsection{Control Law for Underactuated MIMO Systems}

Therefore, in order to handle underactuated, the following transformation is proposed:

$$
Y_{m}=[T] x_{p}
$$

where $Y_{m}$ represents the states of the new coordinate system, $[T]$ is the transformation matrix, whose dimensions are equal to $[B]^{\prime}$. By using Eq. (13), the following updated invertible control input equation is obtained:

$$
u_{y_{m}}=[[T][B]]^{-1}\left[-\lambda\left(\dot{y}_{m}-\dot{y}_{d_{m}}\right)-\left(\ddot{y}_{m}-\ddot{y}_{d_{m}}\right)-\eta \operatorname{sgn}\left(s_{y_{m}}\right)\right]+u_{y_{m_{k-1}}}-\varepsilon_{y_{m}}
$$


where $u_{y_{m}}, s_{y_{m}}, \varepsilon_{y_{m}}$ are the control input matrix, sliding surface, and the control input error in the new coordinate system, respectively. All $y$ terms are obtained using Eq. (13), and $u_{y_{m}}=[T]^{\prime} u_{m}$.

In the cases of non-unitary control input gains, although the exact gain values are not required, an estimate of the bounds of the control input gains on all I/O paths are.

\subsection{Switching Gain}

Eq. (14) is redefined as:

$$
\hat{u}_{y_{m}}=[[T][\hat{B}]]^{-1}\left[-\lambda\left(\dot{y}_{m}-\dot{y}_{d_{m}}\right)-\left(\ddot{y}_{m}-\ddot{y}_{d_{m}}\right)-K_{m} \operatorname{sgn}\left(s_{y_{m}}\right)\right]+u_{y_{m_{k-1}}}-\hat{\varepsilon}_{y_{m}}
$$

where $[\hat{B}]$ is the control input gain estimate matrix, computed using the equation $\hat{B}=\sqrt{b_{\text {upper }} \cdot b_{\text {lower }}}$, and $K_{m}$ is the switching gain. To ensure close-loop stability during the reaching phase, the sliding condition in Eq. (8) is combined with Eq. (9):

$$
s_{m} \dot{s}_{m} \leq-\eta\left|s_{m}\right|
$$

Using Eq. (16), the switching gain for a $2^{\text {nd }}$-order system is obtained as follows:

$$
K_{m}=|\beta-1|\left|\ddot{y}_{m}-\ddot{y}_{d_{m}}\right|+\lambda|\beta-1|\left|\dot{y}_{m}-\dot{y}_{d_{m}}\right|+\left|\hat{B}\left(\sigma_{u}\left(\hat{\varepsilon}_{y_{m}}\right)\right)\right|+\beta \eta
$$

where $\beta=\sqrt{b_{\text {upper }} / b_{\text {lower }}}$.

\subsection{Boundary Layer}

Due to the discontinuity in the control law, high frequency chattering is observed in the controller. Therefore, a smoothing boundary layer, which acts as a low pass filter, is introduced. To assert the attractiveness of the boundary layer, Eq. (16) is updated to the following:

$$
\left|s_{m}\right| \geq \varphi_{m} \rightarrow \frac{1}{2} \frac{d}{d t} s_{m}^{2} \leq\left(\dot{\varphi}_{m}-\eta\right)\left|s_{m}\right|
$$

where $\varphi_{m}$ is the boundary layer thickness. Eq. (18) guarantees that the distance to the boundary layer is always decreasing. The switching gain is then updated to include the boundary layer:

$$
\bar{K}_{m}=K_{m}-\varphi_{m}
$$

which results in the following control law:

$\hat{u}_{y_{m}}=[[T][\hat{B}]]^{-1}\left[-\lambda\left(\dot{y}_{m}-\dot{y}_{d_{m}}\right)-\left(\ddot{y}_{m}-\ddot{y}_{d_{m}}\right)-\bar{K}_{m} \operatorname{sat}\left(s_{y_{m}} / \varphi_{m}\right)\right]+2 u_{y_{m_{k-1}}}-u_{y_{m_{k-2}}}$

where $\operatorname{sat}\left(s_{y_{m}} / \varphi_{m}\right)$ is the saturation function, defined as: :

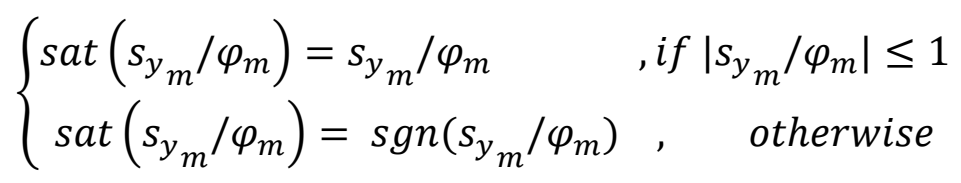


Finally, the time-varying boundary layer thickness is defined using the following differential equation:

$$
\dot{\varphi}_{m}+\lambda \varphi_{m}=K_{m}
$$

where $\varphi_{m}(0)=\eta / \lambda$.

\section{Simulation}

The developed model-free controller was simulated on a couple of nonlinear MIMO systems to illustrate its applicability. The first is a $2^{\text {nd }}$-order nonlinear 2 mass-spring-damper system with 1 input and 2 outputs. The second is the model of a quadrotor with 4 inputs and 6 outputs.

All simulations were performed using Simulink and MATLAB, using the ode5 (Dormand-Price) solver, with a fixed sample time of 0.0001 seconds, and the controller parameters $\lambda=20, \eta=0.1$, and $\sigma_{u}=0.5$.

\subsection{Nonlinear 2 Mass-Spring-Damper System}

The 2 mass-spring-damper system with nonlinear elasticity has the following mathematical model:

$$
\begin{gathered}
m_{1} \ddot{x}_{1}=k_{2}\left(x_{2}-x_{1}\right)-b_{2}\left(x_{2}-x_{1}\right)^{3}+c_{2}\left(\dot{x}_{2}-\dot{x}_{1}\right)-k_{1} x_{1}+b_{1} x_{1}{ }^{3}-c_{1} \dot{x}_{1} \\
m_{2} \ddot{x}_{2}=u-k_{2}\left(x_{2}-x_{1}\right)+b_{2}\left(x_{2}-x_{1}\right)^{3}-c_{2}\left(\dot{x}_{2}-\dot{x}_{1}\right)
\end{gathered}
$$

where the masses are $m_{1}=10 \mathrm{~kg} \& m_{2}=20 \mathrm{~kg}$, the spring constants are $k_{1}=3 \mathrm{~N} / \mathrm{m} \& k_{2}=7 \mathrm{~N} / \mathrm{m}$, the spring stiffening coefficients are $b_{1}=-1.5 \mathrm{~N} / \mathrm{m}^{3} \& b_{2}=-3 \mathrm{~N} / \mathrm{m}^{3}$, meaning the springs become increasingly stiffer as they are elongated, the damping coefficients are $c_{1}=5 \mathrm{~N} / \mathrm{m} \cdot \mathrm{s}^{-1} \& c_{2}=8 \mathrm{~N} / \mathrm{m} \cdot \mathrm{s}^{-1}$, and $u$ is the control input acting on the second mass.

The control input gain matrix is as follows:

$$
B=\left[\begin{array}{c}
0 \\
1 / m_{2}
\end{array}\right]
$$

However, assuming the mass is not exactly known, an estimate for $m_{2}$ is given an upper limit at $25 \mathrm{Kg}$ and $15 \mathrm{Kg}$. The $T$ matrix is therefore given as:

$$
T=\left[\begin{array}{l}
T_{11} \\
T_{21}
\end{array}\right]^{\prime}
$$

where $T_{11}$ weighs $x_{1}$ more heavily and $T_{21}$ weighs $x_{2}$. The desired signal was chosen to be $x_{d}=\sin (\pi t / 2)$. The simulation was performed twice, once with $T=\left[\begin{array}{ll}1.25 & 0.02\end{array}\right]$ and a second time with $T=\left[\begin{array}{ll}0.02 & 1.25\end{array}\right]$, for 20 seconds, and the following results were obtained:
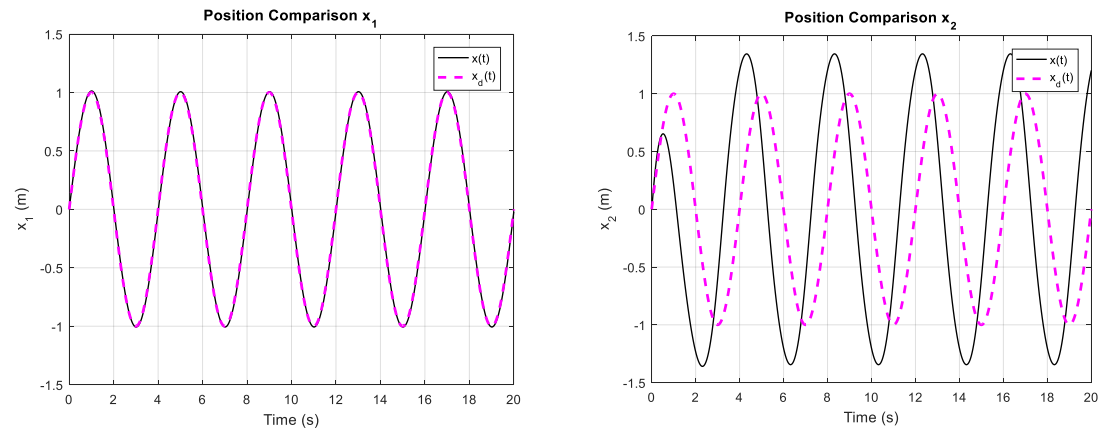

Fig. 1: Plots of $x_{1}$ and $x_{2}$ with the desired signal $x_{d}$. 
Figure 1 shows the output of the system on both mass 1 and 2. As expected, with a $T=\left[\begin{array}{ll}1.25 & 0.02\end{array}\right], x_{1}$ displays perfect tracking, since it is heavily weighted in comparison to $x_{2}$, which does not track the desired signal.
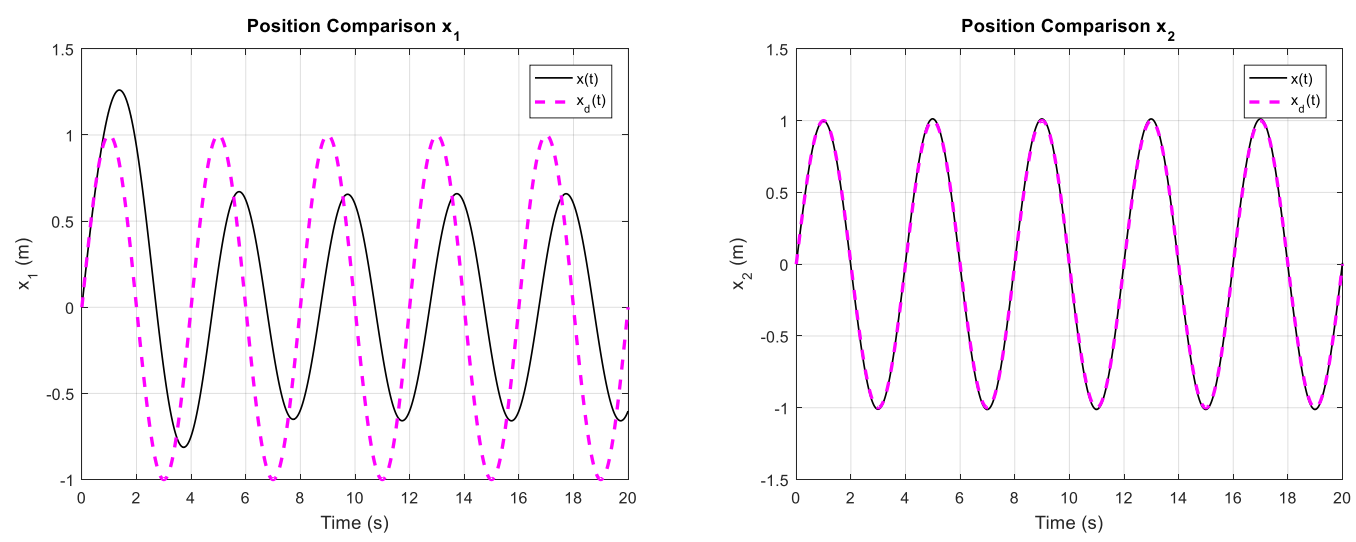

Fig. 2: Plots of $x_{1}$ and $x_{2}$ with the desired signal $x_{d}$.

Figure 2 shows the output of the system on both mass 1 and 2 in case 2 , where $T=\left[\begin{array}{ll}0.02 & 1.25\end{array}\right]$. Therefore, $x_{2}$, being more heavily weighted, displays perfect tracking, while $x_{1}$ does not.
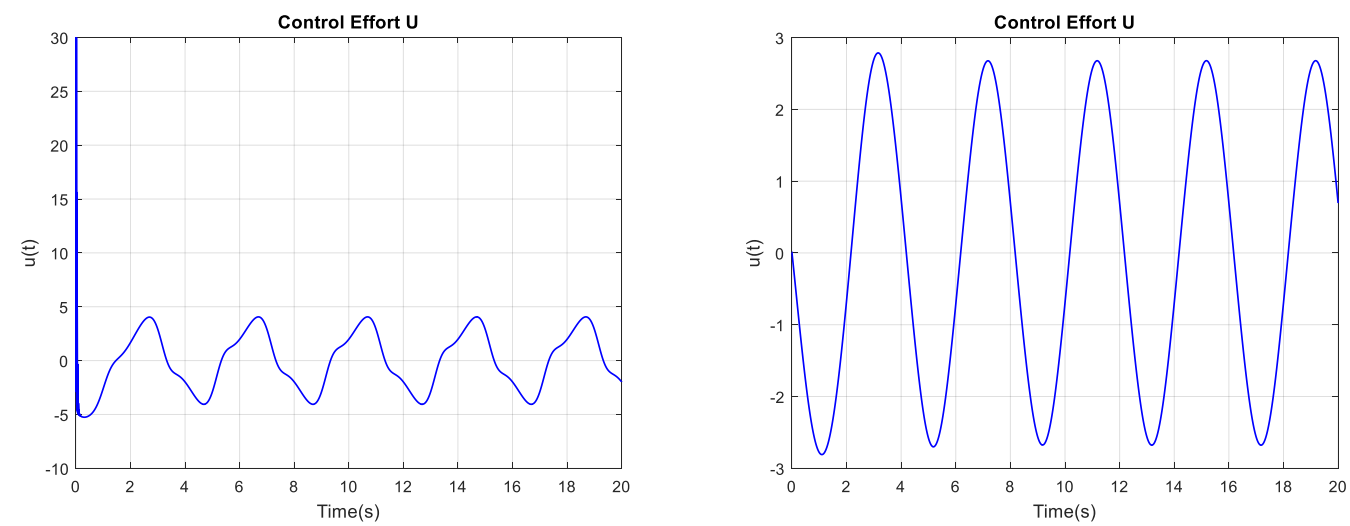

Fig. 3: Plots of $u(t)$. The left plot shows the control effort in case 1. The right plot shows case 2 .

Figure 3 shows the control effort in both cases. It is clear that since the control input acts on mass 2, there is a larger initial spike in the control effort in case 1.

\subsection{Simulation of Quadrotor Position Control}

The second simulation was performed on the model of a quadrotor, a 4-input 6-output system. The model-free controller was used to obtain perfect position tracking of the quadrotor in $x-y-z$. The mathematical model used was obtained from Xu et al. in [3], divided into two subsystems, a fully-actuated subsystem:

$$
\left[\begin{array}{c}
\ddot{z} \\
\ddot{\varphi}
\end{array}\right]=\left[\begin{array}{c}
u_{1}(\cos \varphi \cos \psi)-g \\
u_{4}
\end{array}\right]+\left[\begin{array}{l}
-K_{3} \dot{z} / m \\
-K_{6} \dot{\varphi} / I_{3}
\end{array}\right]
$$

and an underactuated subsystem: 


$$
\begin{gathered}
{\left[\begin{array}{c}
\ddot{x} \\
\ddot{y}
\end{array}\right]=\left[\begin{array}{cc}
u_{1} \cos \varphi & u_{1} \sin \varphi \\
u_{1} \sin \varphi & -u_{1} \cos \varphi
\end{array}\right]\left[\begin{array}{c}
\sin \theta \cos \psi \\
\sin \psi
\end{array}\right]+\left[\begin{array}{c}
-K_{1} \dot{x} / m \\
-K_{2} \dot{y} / m
\end{array}\right]} \\
{\left[\begin{array}{c}
\ddot{\theta} \\
\ddot{\psi}
\end{array}\right]=\left[\begin{array}{l}
u_{2} \\
u_{3}
\end{array}\right]+\left[\begin{array}{c}
-K_{4} \dot{\theta} / I_{1} \\
-K_{5} \dot{\psi} / I_{2}
\end{array}\right]}
\end{gathered}
$$

where $(x, y, z)$ are the position coordinates, $(\theta, \psi, \varphi)$ are the three Euler angles, representing pitch, roll, and yaw, respectively, $K_{i}$ 's are drag coefficients, $g$ is the acceleration of gravity, $l$ is half the length of the quadrotor, and $I_{i}$ 's are the moments of inertia with respect to each axis.

The application of the model-free SMC system to the system in Eq. (28) is similar to that in [8], while the $T$ matrix in this case is a $2 x 4$ matrix. The table of parameters used is shown below, followed by the simulation results:

Table 1: Parameters used in the simulation.

\begin{tabular}{|c|c|c|c|}
\hline \multicolumn{2}{|c|}{ System Parameters } & \multicolumn{2}{c|}{ Desired Conditions } \\
\hline$I_{1}, I_{2}$ & $1.25 \mathrm{Ns}^{2} / \mathrm{rad}$ & $x_{d}$ & $1 \mathrm{~m}$ \\
\hline$I_{3}$ & $2.5 \mathrm{Ns}^{2} / \mathrm{rad}$ & $y_{d}$ & $1 \mathrm{~m}$ \\
\hline$K_{1}, K_{2}, K_{3}$ & $0.010 \mathrm{Ns} / \mathrm{m}$ & $z_{d}$ & $3 \mathrm{~m}$ \\
\hline$K_{4}, K_{5}, K_{6}$ & $0.012 \mathrm{Ns} / \mathrm{rad}$ & $\varphi_{d}$ & $\pi / 3$ \\
\hline$m$ & $2 \mathrm{~kg}$ & \multicolumn{2}{|}{} \\
\hline$l$ & $0.2 \mathrm{~m}$ & \multicolumn{2}{|}{} \\
\hline$g$ & $9.8 \mathrm{~m} / \mathrm{s}$ & \multicolumn{2}{|}{} \\
\hline
\end{tabular}
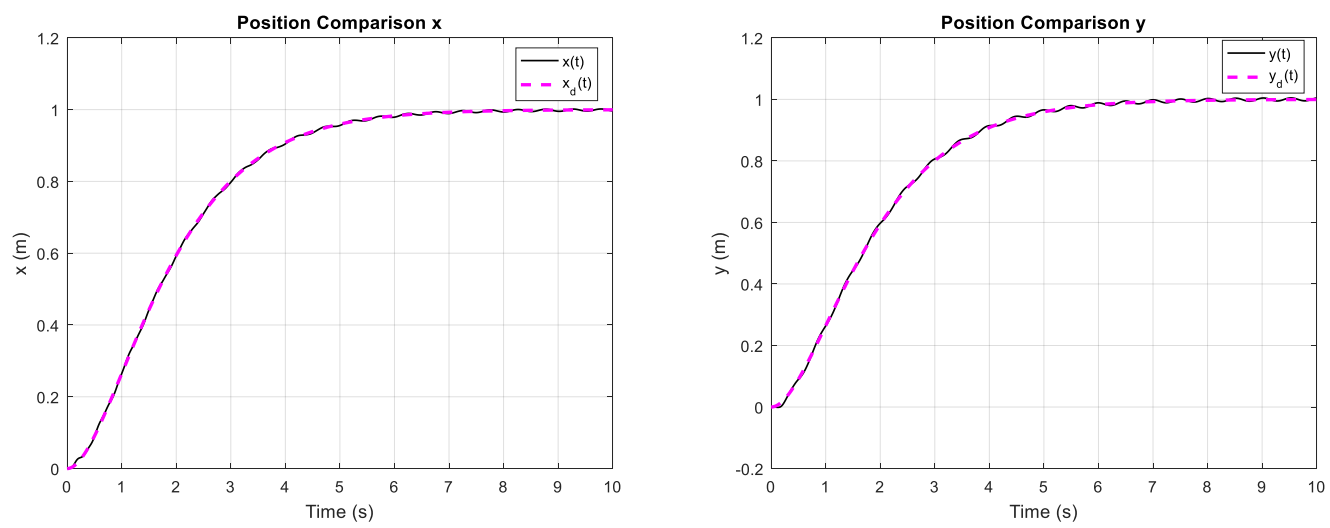

Fig. 4: Plots the position of the quadrotor compared to the desired trajectory.
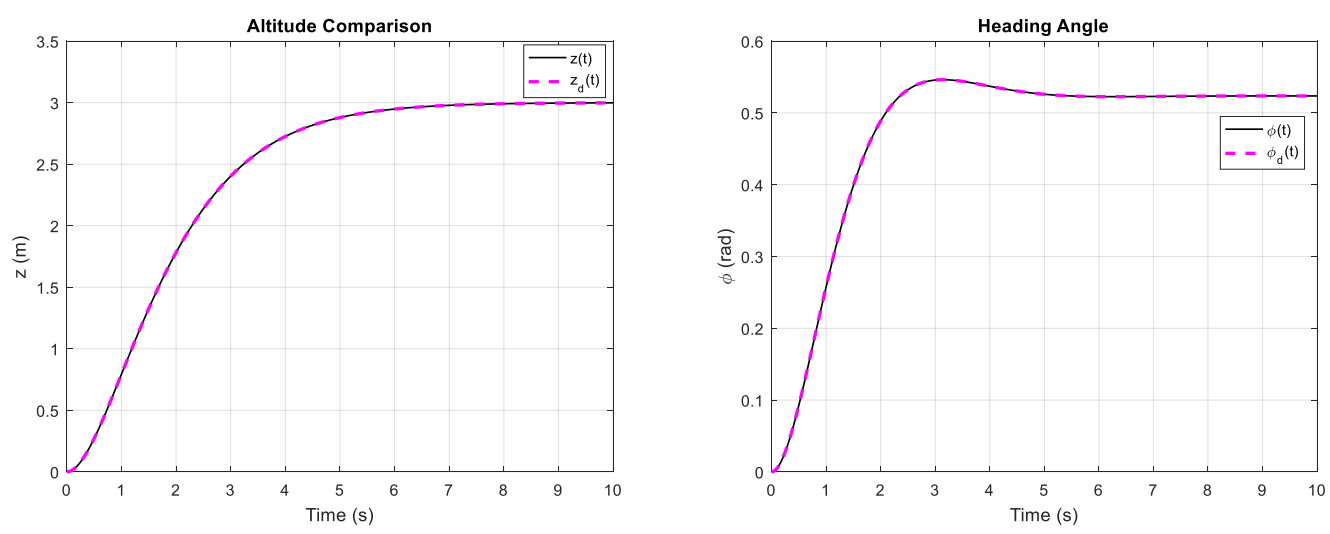

Fig. 5: Plot of the altitude of the quadrotor on the left and heading on the right. 

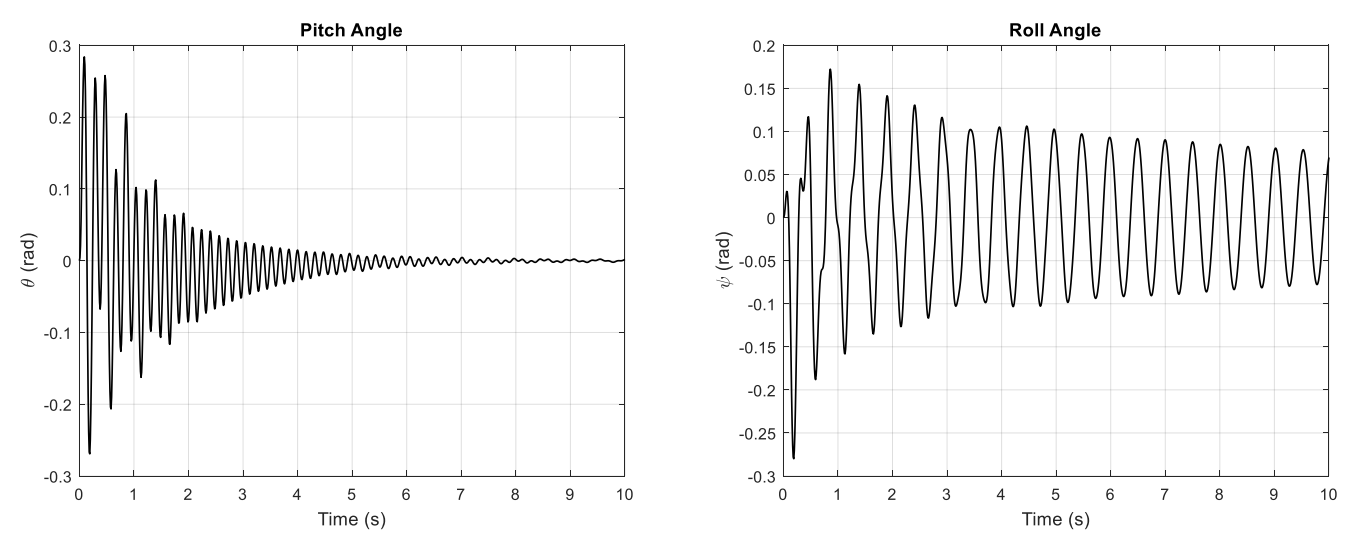

Fig. 6: Plot of the pitch angle on the left and roll angle on the right.

As shown in the figures above, the controller was able to provide perfect tracking of the desired trajectories. One thing to note though is that since the desired trajectory is the complete path from position 0 to $1 \mathrm{~m}$ in both $x$ and $y$, the controller is pretty aggressive in pitching and rolling the quadrotor, causing high frequency activity in both those terms, as shown in Figure 6.

\section{Conclusion}

A model-free sliding mode controller was proposed for MIMO systems. The focus of the paper was on underactuated MIMO systems, which typically represent most systems in practice. By using an algebraic transformation into a different coordinate matrix, the developed control law was applicable to underactuated systems, where elements of the transformation matrix weighted certain states more heavily than others. Finally, the controller was applied on two wellknown systems; a nonlinear 2 mass-spring-damper system with 1 input and a quadrotor model.

\section{References}

[1] J. J. Slotine and W. Li, "Sliding Control," in Applied Nonlinear Control, Englewood Cliffs, NJ: Prentice-Hall, 1991, pp. 276-310.

[2] K. Runcharoon and V. Srichatrapimuk, "Sliding Mode Control of Quadrotor," in Technological Advances in Electrical,Electronics, and Computer Engineering, Konya, Turkey, 2013, pp. 552-556.

[3] R. Xu and U. Ozguner, "Sliding Mode Control of a Class of Underactuated Systems," in Automatica, vol. 44, pp. 233-241, 2008.

[4] R. Olfati-Saber, "Normal Forms for Underactuated Mechanical Systems with Symmetry," in IEEE Transactions on Automatic Control, vol. 47, no. 2, pp. 305-308, 2002.

[5] A. J. Munoz-Vazquez, V. Parra-Vega, and A. Sanchez, "A Passive Velocity Field Control for Naviagation of Quadrotors with Model-free Integral Sliding Mode Control," in Journal of Intellegent \& Robotic Systems, vol. 73, pp. 373-385, 2014.

[6] A. Crassidis and A. Mizov, "A Model-Free Control Algorithm Derived Using the Sliding Mode Control Method," in Proceedings of the 2nd International Conference of Control, Dynamic Systems, and Robotics, Ottawa, ON, 2015.

[7] A. Crassidis and R. Reis, "Model-Free Sliding Mode Control Method," in Proceedings of the 3nd International Conference of Control, Dynamic Systems, and Robotics, Ottawa, ON, 2016. 